\title{
Future of the NOSAMS Tandetron AMS System
}

\author{
Karl F. von Reden* \\ National Ocean Sciences AMS Facility, \\ Department of Geology \& Geophysics, \\ Woods Hole Oceanographic Institution, \\ Woods Hole, MA 02543, U.S.A.
}

\begin{abstract}
The NOSAMS 3 MV Tandetron AMS system will soon reach its $20^{\text {th }}$ anniversary of operation. A critical review of all aspects of the system is necessary to assess the analytical, economic, and operational factors that enter into the consideration of either an upgrade or a replacement of the instrument. Recent in-house experience with the design and construction of a large-acceptance ${ }^{14} \mathrm{C}$ AMS system gives us a benchmark with which to compare the expected outcome of an upgrade. The present ion analysis efficiency of our Tandetron system is roughly $1 \%$, well below the $4 \%$ value of our new system. This affects the lower limit of analyzable sample size, speed of analysis, exposure to systematic errors, and the upper limit of achievable single-sample counting statistics. At first glance it may seem obvious that a replacement (even at higher cost) would be preferable to an upgrade. However, it will be shown that there are good reasons to consider the latter. One of the most compelling is the fact that the Tandetron is a state-of-the-art and superior high-current accelerator design with minimal signs of aging and very low maintenance. Ion beam modeling calculations show that the main transmission limitation of the instrument is caused by the design of our injector, a four-magnet "Recombinator", making it difficult to perform reliable AMS with negative currents exceeding $80 \mu \mathrm{A}$. To be acceptable, an upgrade should bring the Tandetron to at least $3 \%$ ion efficiency by replacing the injector with a properly matched high-transmission device.
\end{abstract}

PACS: 29.27.Ac; 02.70.Dh; 29.27.Eg; 29.30.Aj

Keywords: Accelerator beam optics modeling; FEM; AMS efficiency and acceptance

*Correspondence: kvonreden@whoi.edu, 1-508-289-3384, 1-508-457-2183 (fax)

\section{Introduction}

The US-AMS Corporation 3 MV Tandetron AMS system [1] has been operating successfully at the NOSAMS Facility since the early 1990s. The system is designed for radiocarbon analyses only and is equipped with a "recombinator" injector [2] for simultaneous injection and analysis of the three carbon isotope beams. The core of the system, the accelerator is essentially state-ofthe-art and it has been truly reliable and low-maintenance, requiring tank openings only rarely (3-5 years) to inspect/replace drive shaft bearings and stripper gas recirculation pump. We feel that an upgrade around this accelerator is well worth the effort. Many years of data acquisition have resulted in the determination that the system is producing good AMS data with ion beams 
$11^{\text {th }}$ International Conference on Accelerator Mass Spectrometry, Rome, September 2008 Manuscript \#41

up to $-80 \mu \mathrm{A}\left({ }^{12} \mathrm{C}\right)$ at a source emittance of less than $20 \pi \mathrm{mm}$ mrad (in laboratory units). We have established that the extraction electrode geometry constraining the source to this low emittance also limits its efficiency. The measured AMS ion efficiency of our system (C ions analyzed/C atom content of sample with good AMS results) is approximately 1\%. Modern highcurrent sputter ion sources can produce ion beam of several hundred $\mu \mathrm{A}$ but their source emittance at these currents is closer to $40 \pi \mathrm{mm}$ mrad. AMS systems designed with large acceptance reach over $4 \%$ ion efficiency [3]. We are currently working to reach that level with our new compact system [4].

A major trend in AMS over the last decade has been to increase the capability of measuring ever smaller samples, while also trying to achieve higher precision on any size of sample. Systems with low ion efficiency will have a difficult time keeping up with this trend.

Our first attempt to upgrade the system in the late 1990s with a high-current ion source to reduce measurement time and boost throughput faltered because of the limitations described above. We then considered options to upgrade the recombinator to increase its acceptance but soon realized that the main cause of its transport limitation is the unavoidable focal plane rotation at the exit of such an arrangement of magnets. The foci of the three carbon beams are separated by several $\mathrm{cm}$ in beam direction, leading to a compromise in the match to the low energy accelerator column that the "Q-snout" lens in the entrance of the column in our system cannot fully overcome. The following discussion is based on the assumption that the only other significant acceptance limitation in our system is the gas stripper canal in the terminal of the Tandetron. 
$11^{\text {th }}$ International Conference on Accelerator Mass Spectrometry, Rome, September 2008 Manuscript \#41

\section{Calculations}

The purpose of this paper is to examine whether the Tandetron will accept and transport beams from a high-current source using a single magnet sequential injector. The ion optical "bottleneck" of any AMS system with gas stripping is the stripper canal in the high voltage terminal of the accelerator. Model calculations of our current setup with a recombinator injector show that the canal is the acceptance limitation in our system. Beams that clear the canal will have no further restriction in the remainder of the system (if evacuated properly). For the calculations presented here we replace the injector with modified versions of a MC-SNICS [5, 6] and a Danfysik [7] double-focusing $90^{\circ}$ magnet (68 mm gap) and remove the "Q-snout” lens in the entrance of the accelerator. The starting point of this exercise is a realistic representation of the extracted ion beam from a MC-SNICS source. Using code PBGUNS [8] the laboratory emittance was calculated for a $60 \mathrm{keV} 285 \mu \mathrm{A} \mathrm{C} \mathrm{C}^{-}$beam at the exit of the source (fig. 1). The code calculates the extracted negative ion beams (allowing multiple particle masses) in the presence of the modified extraction potential due to the space charge of the intense $\mathrm{Cs}^{+}$sputter beam. Over 600 particles are modeled in the extracted beam. A subset of 33 (R, R') pairs was selected to represent the ion beam in subsequent ray tracing calculation with finite-element method (FEM) modeling codes MagNet and ElecNet [9]. For realistic calculations a maximum 3D mesh size of $(5 \mathrm{~mm})^{3}$ was forced in the inner part of the beam line, particularly important in the fringe field regions of the beam optics devices. The time resolution for the trajectory calculations was $1 \mathrm{~ns}$, resulting in 2000 steps per ion optics element. The initial rays were limited to the horizontal, vertical, and $\pm 45^{\circ}$ planes of the layout, a reasonable representation of the solid angle.

An example of the detail of the calculated fields is shown in figure 2 for the injector magnet. The mesh is superimposed on the graph that shows the center plane B field in the magnet. The ion 
$11^{\text {th }}$ International Conference on Accelerator Mass Spectrometry, Rome, September 2008 Manuscript \#41

beam exiting on the upper right is drifting to the object point of the low-energy accelerator tube.

The insert in figure 2 shows a vertical slice of the magnet in the fringe field region. Note that the beam diameter inside the magnet reaches more than $20 \mathrm{~mm}$ (30\% of the gap size). This is the main reason for choosing a large pole gap to avoid aberrations.

Our present system requires an emittance matching lens at the entrance of the accelerator to overcome the strong focusing effect of the initial acceleration gap. This "Q-snout", an asymmetric three-cylinder lens with $25 \mathrm{~mm}$ diameter and 0.2 gap-to-diameter ratio is geometrically too small to handle large beams without serious aberration. By raising the beam energy from our presently used value of about $40 \mathrm{keV}$ to $60 \mathrm{keV}$ we avoid the need of a matching lens if the first three electrode gaps in the accelerator are operated at reduced gradient.

We pick up the ray trajectories at the object point of the low-energy accelerator tube and follow their fate to the accelerator terminal and the stripper canal (1.2 m long, $12.7 \mathrm{~mm}$ diameter) contained in it. Figure 3 shows the result of the calculation for a tube with parallel electrodes. The beam clears the stripper canal with room to spare.

\section{Complication}

The upgrade decision would be straight forward, given the results so far. However, the original design of our Tandetron included spirally inclined electrodes for electron suppression in the lowenergy tube. The effect of inclined electrodes cannot be calculated with traditional transport codes that treat accelerator sections as boxes with cylindrical symmetry. Fine-mesh FEM will allow the modeling of such an electrode arrangement. The modeled [10] geometrical setup of the low energy tube electrodes is shown in the schematic diagram in figure 4. The basic electrode 
$11^{\text {th }}$ International Conference on Accelerator Mass Spectrometry, Rome, September 2008 Manuscript \#41

gap size is $2.54 \mathrm{~cm}$, the gradient $12.2 \mathrm{kV} / \mathrm{cm}$. In addition to the electrode inclination $\left(7.5^{\circ}\right)$ small permanent magnets are mounted on the electrodes for increased electron suppression. Figure 5 shows the effect of the spiral electrode inclination on the low energy acceleration stage beam transport in a detailed section plot of the low-energy tube and the stripper canal. The 0.1 scaling in beam direction makes it difficult to see the angular inclination or rotation of the electrodes. It is apparent that the path length of the trajectories is increased due to the spiraling motion of the particles and that the beam becomes larger in both diameter and angular divergence. However, the calculation suggests that the beam still clears the stripper canal and is actually comparable in divergence to the result of the original design calculations with code RAYTRACE [11] for the transmission of much smaller ion beams injected through the recombinator.

\section{Conclusions}

Tandetrons are known to be capable of accelerating large beams in non-AMS applications. In such systems some loss of beam is tolerable as fractionation usually does not matter. In AMS applications fractionation is generally undesirable, especially if it is current dependent. However, larger currents are desirable because they reduce the run time/sample and decrease systematic errors due to shorter lag times between standards and unknowns. It has become increasingly important to raise the efficiency in our system as a larger number of our samples are well below the $500 \mu \mathrm{g}$ of carbon, considered "normal" size. Modern AMS systems should be capable of transmitting beams well above $100 \mu \mathrm{A}$ of ${ }^{12} \mathrm{C}^{-}$with ${ }^{14} \mathrm{C}$ count rates of at least $400 / \mathrm{s}$ for modern samples of normal size. Our routine operating conditions currently allow $50-70 \mu \mathrm{A}$ of ${ }^{12} \mathrm{C}^{-}$and

${ }^{14} \mathrm{C}$ rates of 120 - 160/s for modern samples. By replacing the recombinator with a high-current 
$11^{\text {th }}$ International Conference on Accelerator Mass Spectrometry, Rome, September 2008 Manuscript \#41

sequential injector we believe that it is possible to achieve at least a threefold increase in efficiency in our system. Because of the $10-15 \%$ lower stripping yield at charge state 3+ (compared to $1+$ or $4+$ ) we do not expect to match the efficiency of the compact systems or the large tandems. However, another aspect of this upgrade may make our system highly competitive: given our high-energy dipole - deflector - dipole setup, the measured background rates with simultaneous injection are already comparable to the results with sequential injectors.

The removal of the stable isotope currents from the system during the ${ }^{14} \mathrm{C}$ acquisition phase of a sequential cycle is expected to lower the background rates even more.

In summary, the described upgrade to the NOSAMS Tandetron seems to be a reasonable lowcost alternative to a complete replacement of the system.

\section{Acknowledgment}

This work has been supported by the United States National Science Foundation under

Cooperative Agreements OCE-0228996 and OCE-0753487.

\section{References and notes}

1. K. H. Purser, T. H. Smick, R. K. Purser, Nucl. Inst. Meth. B52 (1990) 263-268; K. F. von Reden, R. J. Schneider, G. J. Cohen, G. A. Jones, Nucl. Inst. Meth. B92 (1994) 7-11; the system is now produced and marketed by High Voltage Engineering Europe, B.V., Amersfoort, NL.

2. A. E. Litherland, L. R. Kilius, Nucl. Inst. Meth. B52 (1990) 375-377.

3. S. J. Fallon, T. P. Guilderson, T. A. Brown, Nucl. Inst. Meth. B259 (2007) 106-110.

4. B. X. Han, K. F. von Reden, M. L. Roberts, R. J. Schneider, J. M. Hayes, W. J. Jenkins, Nucl. Inst. Meth. B259 (2007) 111-117.

5. National Electrostatics Corporation, Middleton, WI, USA.

6. J. R. Southon, G. dos Santos, B. X. Han, Radiocarbon, Vol.49, No.2 (2007) 301-306.

7. Danfysik A/S, Jyllinge, DK; J. R. Southon, personal communication. 
$11^{\text {th }}$ International Conference on Accelerator Mass Spectrometry, Rome, September 2008 Manuscript \#41

8. PBGUNS 5.04, J.E. Boers, Thunderbird Simulations, Garland, TX, USA.

9. Infolytica Corporation, Montréal, Québec, CA.

10. The precise schedule of the electrodes could not be verified from our documentation. Small changes in the arrangement do not significantly affect the result reported here.

11. Computer code RAYTRACE, H. Enge, S. Kowalski, MIT, Cambridge, MA, USA. 
$11^{\text {th }}$ International Conference on Accelerator Mass Spectrometry, Rome, September 2008

Manuscript \#41

\section{Figure captions}

Fig. 1: Calculated emittance plot for a $60 \mathrm{keV}, 285 \mu \mathrm{A}$ ion beam exiting a modified MC-SNICS ion source. Black disks indicate ( $\left.R, R^{\prime}\right)$ coordinates used for rays in the FEM tracing calculations of this study. 33 rays cover the horizontal, vertical, and two $45^{\circ}$ planes of the geometry.

Fig. 2: Example of the detail of the FEM modeling used in this paper. The projection of the 3D mesh is superimposed on the figure. Both shading and contours depict the B field strength in the horizontal and vertical planes. While the shading reflects some smoothing, the contours follow the shape of the tetrahedrical mesh elements. Note the strong B field distortion near the pole edges on the insert depicting a vertical slice in the fringe field section of the magnet. Beams extending into these regions likely will suffer aberration

Fig. 3: Ray tracing results for the low energy acceleration stage with parallel electrodes operated at $12.2 \mathrm{kV} / \mathrm{cm}$ gradient. No matching lens is necessary to transport the beam easily through the $1.17 \mathrm{~m}$ long, $12.7 \mathrm{~mm}$ diameter stripper canal. The figure is scaled in beam direction by 0.01 .

Fig. 4: Arrangement of the electrodes in the spiral inclination section of the low energy acceleration section of the NOSAMS Tandetron. Central distance of the electrodes: $25.4 \mathrm{~mm}$. Gradient: $12.2 \mathrm{kV} / \mathrm{cm}$. The calculated effect of this arrangement on the beam can be seen in figure 5 .

Fig. 5: Detail of the ray tracing result for a low energy acceleration stage with spirally inclined electrodes (only inclined section shown). The $7.5^{\circ}$ tilt of the individual electrodes cannot be seen in this plot due to scaling by 0.1 in beam direction. The resulting spiral deflection of the beam is clearly visible but not big enough to restrict the beam in the stripper canal. 
$11^{\text {th }}$ International Conference on Accelerator Mass Spectrometry, Rome, September 2008 Manuscript \#41

Fig 1.:

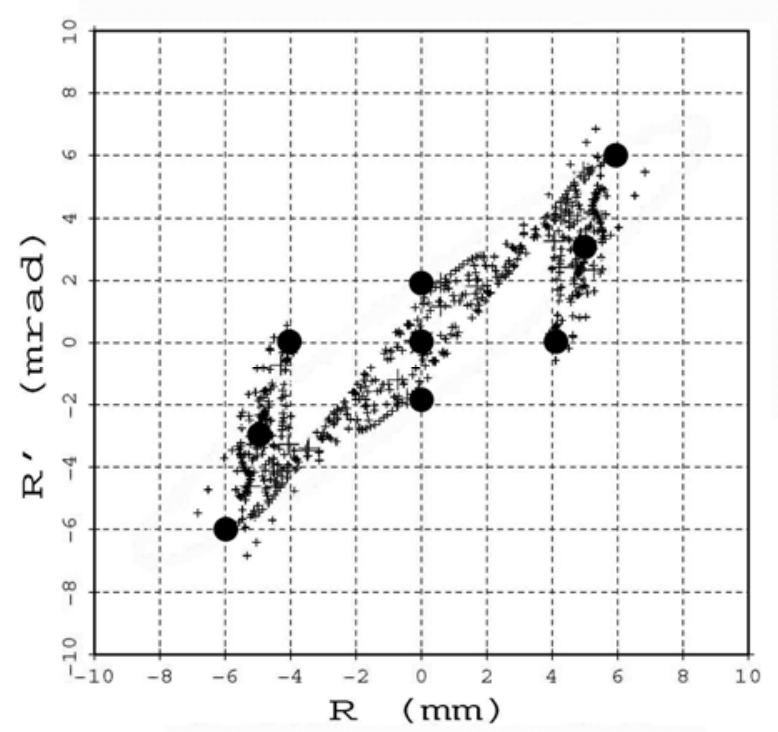

Fig. 2

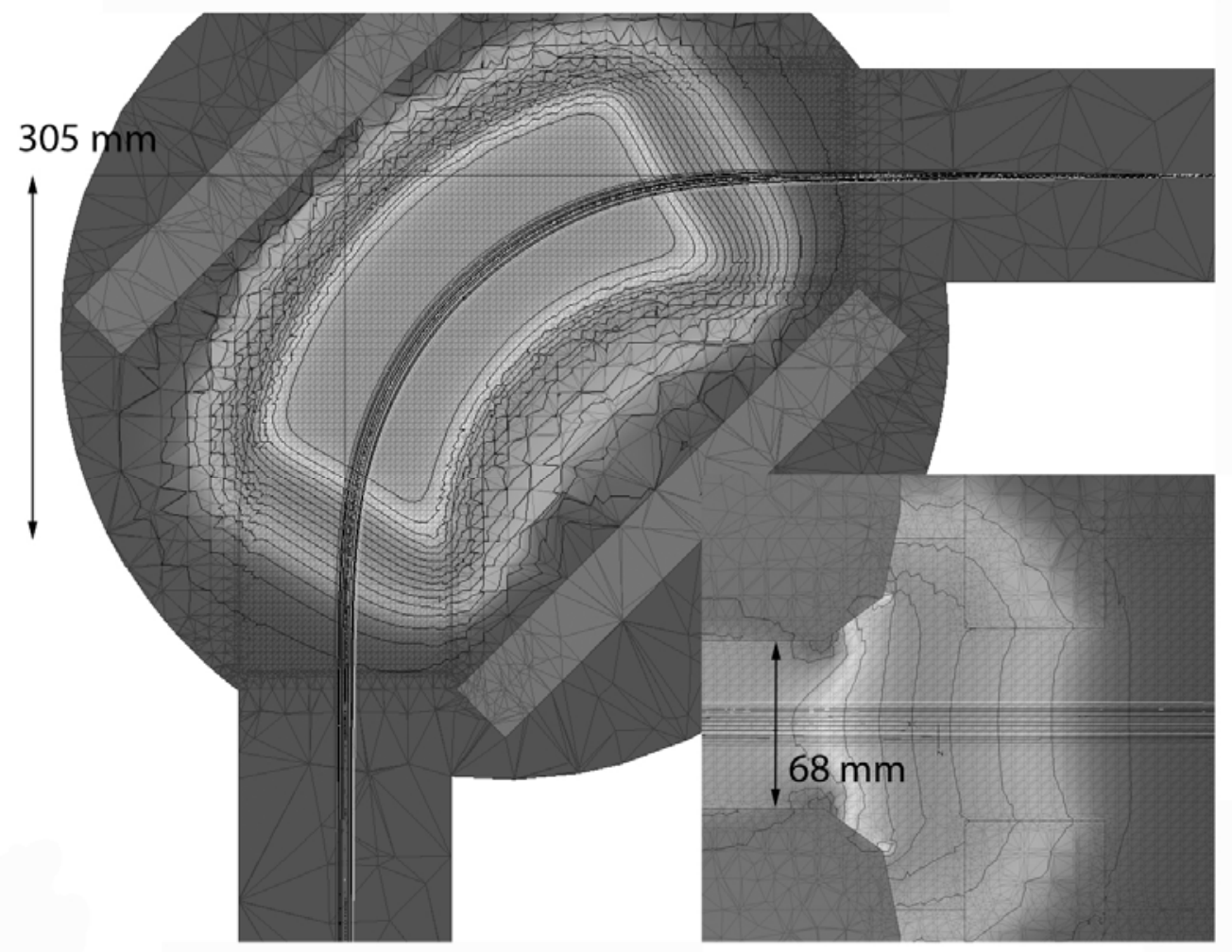


Fig. 3

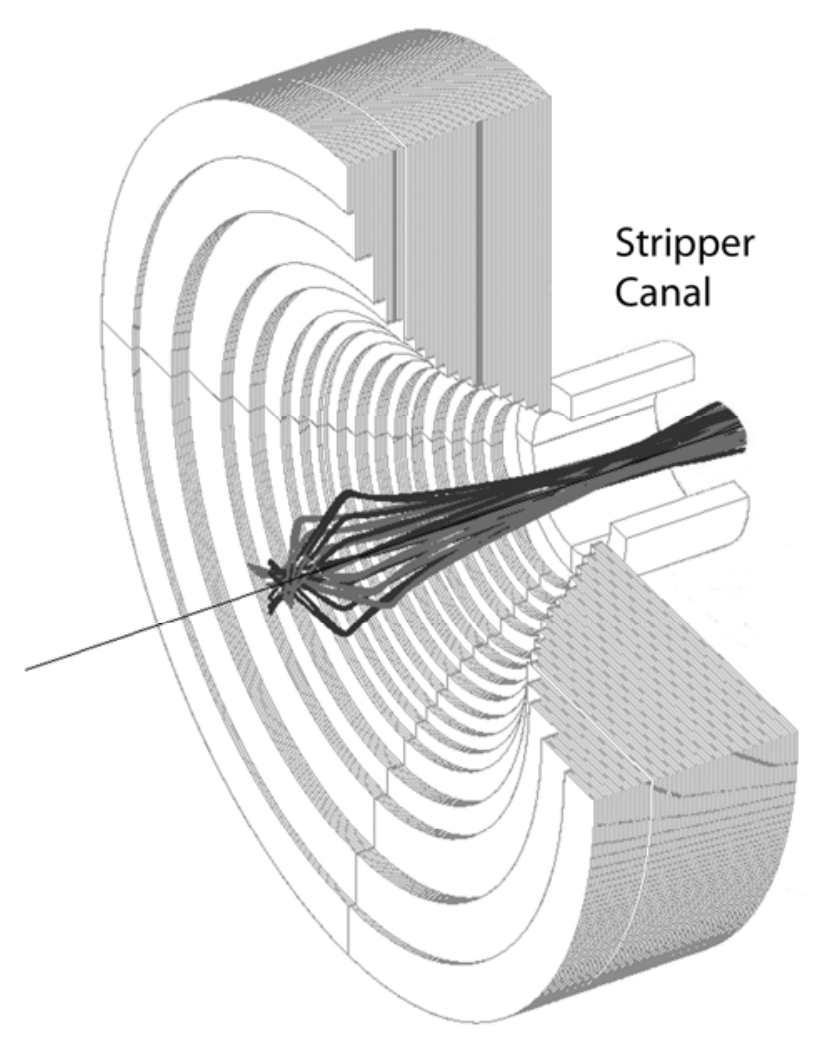

Fig. 4

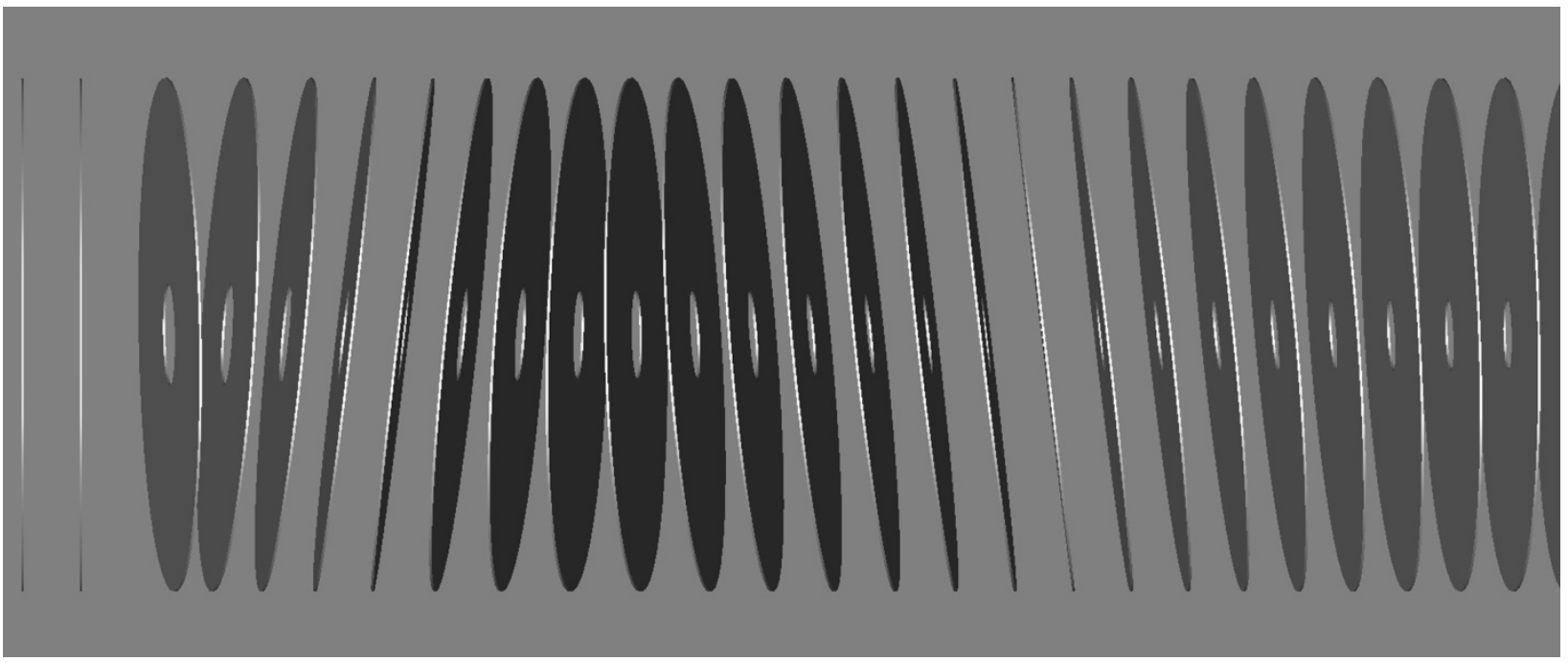


$11^{\text {th }}$ International Conference on Accelerator Mass Spectrometry, Rome, September 2008 Manuscript \#41

Fig. 5

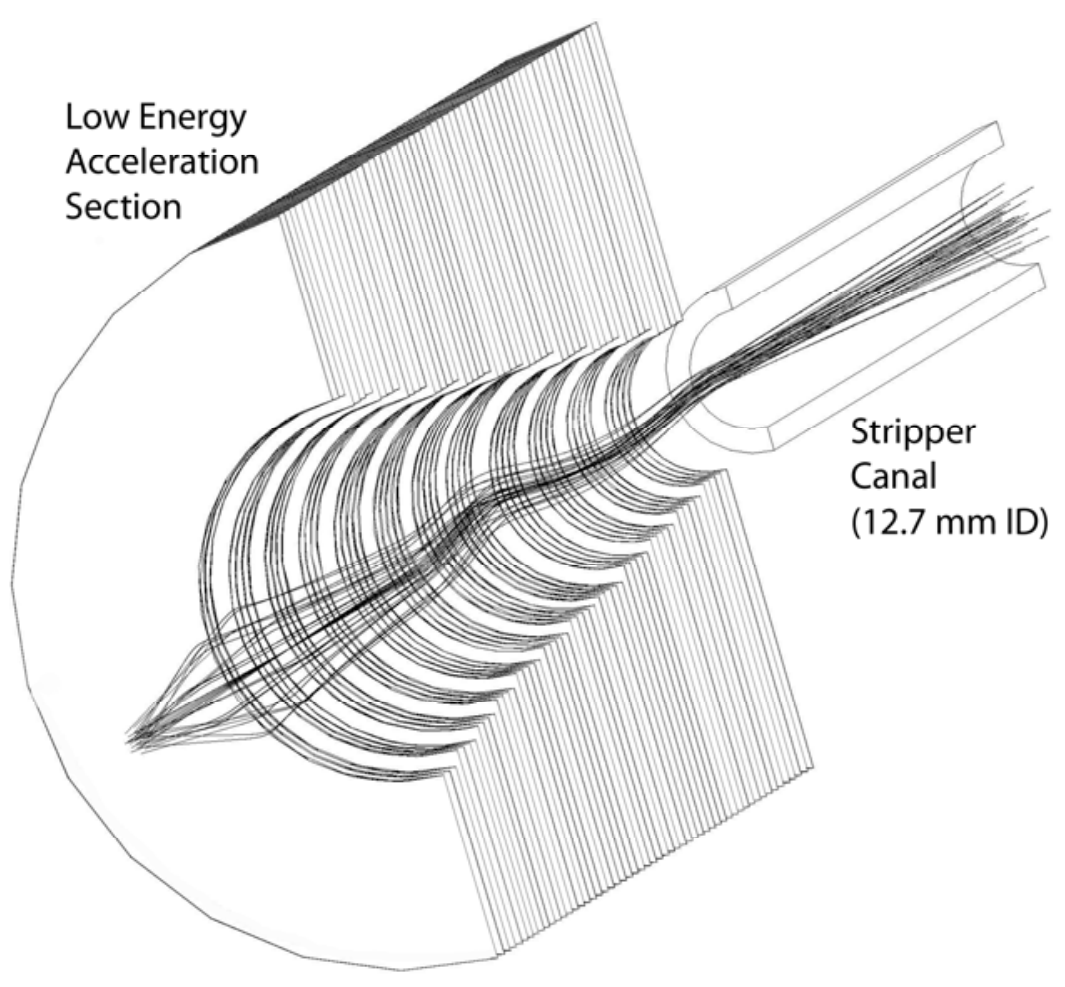

